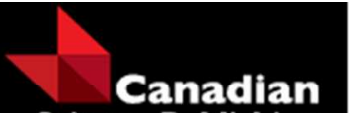
Science Publishing

Canadian Journal of Physiology and Pharmacology Revue canadienne de physiologie et pharmacologie

\title{
A Primer on Current Progress in Cardiac Fibrosis
}

\begin{tabular}{|r|l|}
\hline Journal: & Canadian Journal of Physiology and Pharmacology \\
\hline Manuscript ID & cjpp-2016-0687.R1 \\
\hline Manuscript Type: & Review \\
\hline Complete List of Authors: & $\begin{array}{l}\text { Al Hattab, Danah; St Boniface Hospital Albrechtsen Research Centre, } \\
\text { Physiology and Pathophysiology } \\
\text { Czubryt, Michael; University of Manitoba, Department of Physiology and } \\
\text { Pathophysiology, Rady Faculty of Health Sciences, R4008 St Boniface } \\
\text { Hospital Albrechtsen Research Centre }\end{array}$ \\
\hline Keyword: & $\begin{array}{l}\text { cardiac fibrosis, extracellular matrix, fibrosis therapy, fibroblast, } \\
\text { myofibroblast }\end{array}$ \\
\hline &
\end{tabular}

\section{SCHOLARONE ${ }^{\text {Tw }}$}

Manuscripts 


\title{
A Primer on Current Progress in Cardiac Fibrosis
}

\author{
Danah Al Hattab and Michael P. Czubryt ${ }^{*}$
}

Department of Physiology and Pathophysiology, Rady Faculty of Health Sciences, University of Manitoba, Winnipeg, Manitoba, Canada

*Address for correspondence:

R4008 St Boniface Hospital Albrechtsen Research Centre

351 Tache Avenue

Winnipeg, Manitoba, Canada

R2H $2 \mathrm{~A} 6$

Tel. 1-204-235-3719

Email: $\underline{\text { mczubryt } @ \text { sbrc.ca }}$

Keywords: cardiac fibrosis; extracellular matrix; fibrosis therapy; fibroblast; myofibroblast 


\begin{abstract}
Cardiac fibrosis is a significant global health problem that is closely associated with multiple forms of cardiovascular disease, including myocardial infarction, dilated cardiomyopathy and diabetes. Fibrosis increases myocardial wall stiffness due to excessive extracellular matrix deposition, causing impaired systolic and diastolic function and facilitating arrhythmogenesis. As a result, patient morbidity and mortality are often dramatically elevated compared to those with cardiovascular disease but without overt fibrosis, demonstrating that fibrosis itself is both a pathologic response to existing disease and a significant risk factor for exacerbation of the underlying condition. The lack of any specific treatment for cardiac fibrosis in patients suffering from cardiovascular disease is a critical gap in our ability to care for these individuals. Here we provide an overview of the development of cardiac fibrosis, and discuss new research directions that have recently emerged and that may lead to the creation of novel treatments for patients with cardiovascular diseases. Such treatments would, ideally, complement existing therapy by specifically focusing on amelioration of fibrosis.
\end{abstract}




\section{Introduction}

Tissue fibrosis occurs when synthesis of the collagen-rich extracellular matrix (ECM), primarily by cardiac fibroblasts, occurs at a higher rate than degradation, resulting in net ECM deposition, alterations in tissue structure and frequently, organ dysfunction (Travers et al. 2016). It has been suggested that fibrotic diseases are associated with $45 \%$ of deaths per year in the western world, yet treatment options are conspicuously limited (Wynn 2004). Indeed, at present only two drugs have been approved specifically to treat fibrosis of any kind: pirfenidone and nintedanib are currently available to treat idiopathic pulmonary fibrosis, and of these, only pirfenidone has been tested for cardiac fibrosis in the research setting (Nanthakumar et al. 2015). This gap has led to a significant recent upsurge in interest in both the pathogenesis of fibrosis, and in the development of novel treatments.

While cardiac fibrosis is a hallmark of a broad constellation of pathological conditions, it is also a risk factor for further dysfunction leading to adverse patient outcomes (Figure 1). Fibrosis interferes with both systolic and diastolic function, can lead to arrhythmogenesis, and increases the likelihood of heart failure (Roche et al. 2015). In short, cardiac fibrosis makes a bad situation much worse. Several patterns of cardiac fibrosis presentation have been noted, and while the reasons why patients develop particular patterns remain unclear, mid-wall fibrosis specifically has been reported to be a strong independent predictor of morbidity, including major adverse cardiovascular events, and mortality (Almehmadi et al. 2014). In a recent study, 30\% of patients diagnosed with non-ischemic dilated cardiomyopathy (DCM) developed mid-wall fibrosis as determined by cardiac magnetic resonance imaging (Assomull et al. 2006). DCM patients with mid-wall fibrosis had an 18-fold greater risk of pump failure and sudden cardiac death compared to DCM patients without mid-wall fibrosis (Leyva et al. 2012). A similar study demonstrated the 
clinical significance of cardiac fibrosis in children with Duchenne muscular dystrophy. The prevalence of fibrosis increased with age, concomitant with a decrease in left ventricular ejection fraction, and increasing involvement of septal fibrosis correlated with increased patient morbidity (Hor et al. 2013). At present, there are no clinically-employed treatments specifically aimed at arresting or reducing cardiac fibrosis, although a number of currently employed medications, targeted at other aspects of cardiovascular disease, have shown some beneficial effect. Unfortunately, these effects are typically modest and secondary to correction of underlying cardiovascular disease features, rather than addressing fibrosis directly. Understanding the extra- and intracellular signaling pathways activated in fibrosis is a critical step in the development of anti-fibrosis therapies.

\section{Fibrosis is a Maladaptive Response to Cardiac Dysfunction}

In response to stress or damage, the heart frequently responds poorly. Cardiac hypertrophy, changes in chamber dimensions and fibrosis are among the most commonly observed alterations, all of which can exacerbate cardiac dysfunction (Roche et al. 2015). Interestingly, such responses frequently occur almost regardless of the precipitating factor, suggesting the induction of common stress response pathways, possibly as an attempt to normalize cardiac output. Initially the heart adapts to these changes, leading to a compensated state, but often such alterations lead to further declines in systolic and/or diastolic function, resulting in decompensation and heart failure.

The precise mechanisms linking myocardial fibrosis and heart failure are not yet fully understood. Pathologic remodeling of the heart, including fibrosis, is extensive in heart failure patients. In the later stages of heart disease, the filling or pumping efficacy of the heart becomes 
impaired. There is an increase in not only collagen synthesis, but also in its crosslinking, increasing the stiffness of the myocardium (Querejeta et al. 2004). This extensive fibrotic deposition is associated with many overlapping exacerbating factors such as diabetes and aging, and treatment of fibrosis may attenuate such conditions (Tarone et al. 2014).

After myocardial infarction (MI), which results in wide-scale necrotic loss of cardiomyocytes, cardiac fibrosis arises in two ways. First, the limited regenerative capacity of cardiomyocytes requires the rapid formation of a fibrotic scar (Prabhu and Frangogiannis 2016). This initial reparative fibrosis is crucial as it prevents the rupture of the ventricular wall, although excessive scar expansion can actually impair the mechanical function of the heart (Czubryt 2012). Second, at longer time-points post-MI, an exaggerated fibrotic response extending well beyond the margin of the infarcted border zone occurs, resulting in cardiac stiffening (Talman and Ruskoaho 2016). This secondary fibrosis may arise from altered mechanical stress occurring throughout the heart due to altered vectoring of physical force through the myocardium necessitated by the presence of the large, akinetic collagen-rich scar.

Increased cardiac afterload may be sufficient to result in a state of pressure overload, and is caused by precipitating factors such as cardiac valve dysfunction or hypertension. In response, a robust interstitial fibrosis can develop over time, likely as a response to increased transmural pressure (Czubryt 2012). Concentric hypertrophy of the myocardium and increased interstitial fibrosis can contribute to a reduction in chamber volume and increased myocardial stiffness, impairing the cardiac filling in diastole. Fibrosis will continue to worsen while the underlying cause of the pressure overload persists (Debonnaire et al. 2015).

Besides the impairment of mechanical function that fibrosis exerts on the heart, interstitial fibrosis and the fibrous post-MI scar can interfere with the normal electrical activity of the heart, 
leading to the development of arrhythmias (de Jong et al. 2011). Both the scar and regions of fibrosis, in the form of foci or diffuse, patchy ECM-rich bands, possess different electrical properties from the surrounding cardiomyocytes, behaving as an electrical insulator. Fibrotic areas can thus either slow or block conduction of the action potential, predisposing individuals to potentially fatal arrhythmias and sudden cardiac death. Not surprisingly, cardiac fibrosis is thus an independent risk factor for overall mortality, sudden cardiac death and the requirement for implantation of a cardioverter-defibrillator in heart failure patients (Almehmadi et al. 2014).

Other cardiac disorders such as cardiomyopathies can also have a significant role in contributing to cardiac fibrosis. For example, mutations in sarcomere genes encoding key contractile proteins in cardiomyocytes, such as myosin heavy chain beta 7 (MYH7) and cardiactype myosin binding protein C (MYBPC3), can result in familial hypertrophic cardiomyopathy (Bonne et al. 1998). This cardiomyopathy is a primary genetic disease of cardiomyocytes, and the resulting impairment in normal cardiac function leads to activation of pro-fibrotic signaling pathways, resulting in interstitial fibrosis, arrhythmias and increased risk of sudden cardiac death. Thus, factors that negatively impinge upon normal cardiac function - whether those external to the heart such as hypertension, those intrinsic to the heart such as cardiomyopathies or valve disorders, or those resulting from acute damage such as MI - result in the development of fibrosis that can, in turn, further degrade cardiac function, culminating in heart failure and greatly increased risk of death.

\section{The Contribution of Inflammation to Cardiac Fibrosis}

Inflammation is now recognized as a broad inducer or facilitator of disease processes throughout the body, with much greater impact on pathogenesis and healing than previously 
thought. In the heart, cytokines released by a variety of inflammatory cells can play a significant role in the activation of myofibroblasts, resulting in fibrosis. In addition, the release of myriad proteases by inflammatory cells results in ECM degradation and remodeling. Inflammatory cells, including monocytes, macrophages, lymphocytes and mast cells, are thus important modulators of cardiac fibrosis (Kong et al. 2014). The inflammatory response can also be induced or exacerbated by cardiac endothelial cells, which can recruit monocytes and macrophages via the release of multiple cytokines, chemokines and pro-fibrotic mediators, such as transforming growth factor $\beta$ (TGF $\beta$ ), fibroblast growth factors or endothelin-1 (Wynn 2008).

After MI, numerous inflammatory cells including neutrophils and macrophages infiltrate the injury site, releasing a pool of cytokines such as tumor necrosis factor $\alpha(\mathrm{TNF} \alpha)$, and interleukins (IL) IL-1 $\beta$ and IL-6 $\beta$ (Travers et al. 2016). Pro-inflammatory cytokines upregulated after myocardial infarction can also be released by activated cardiac fibroblasts to facilitate further fibroblast proliferation and expansion of myocardial fibrosis (Travers et al. 2016). In myocarditis or acute inflammatory cardiomyopathy, T-lymphocytes secrete cytokines that modulate collagen synthesis and the expression of matrix metalloproteinases (MMPs) and tissue inhibitors of MMPs (TIMPs), resulting in ECM remodeling (Wei 2011). Inflammatory cells are also a direct source of pro-fibrotic growth factors such as TGF $\beta$, platelet-derived growth factors (PDGF) and fibroblast growth factors, which directly stimulate proliferation of fibroblasts and their conversion to myofibroblasts (Kong et al. 2014). In turn, activated fibroblasts can also serve as an additional source of these growth factors.

In patients with heart failure, elevated levels of "stress-mediated" pro-inflammatory cytokines such as TNF $\alpha$, IL-6, and IL-1 $\beta$ are powerful predictors of poor prognosis. These factors are mainly produced by monocytes and mast cells, as well as cardiomyocytes (Hara et al. 
2002). While the direct involvement of lymphocytes in cardiac fibrosis is incompletely characterized, CD4+ helper cells may up-regulate pro-fibrotic cytokines IL-4 and IL-13, which can work as potent stimulators for fibroblast collagen synthesis in the myocardium (Wynn 2008). Besides monocytes and macrophages, cardiac mast cells have also been implicated in the fibrotic remodeling of the heart by playing a significant role in the production various types of fibrotic mediators, growth factors, vasoactive agents and cytokines that are capable of mediating cardiac remodeling (Batlle et al. 2007).

Cardiac transplantation is often a strategy of last resort for the treatment of heart failure patients, however cardiac allograft rejection due to activation of an anti-graft immune response can also lead to cardiac fibrosis and vasculopathy. Graft rejection is characterized by basophilmediated release of cytokines that activate the donor-derived fibroblasts to produce an excessive amount of ECM (Pichler et al. 2012; Schiechl et al. 2016). Patients with late-failing cardiac allografts exhibit occlusive narrowing of the coronary vessels and progressive replacement fibrosis of the parenchyma, which devolves over time to induce cardiac dysfunction (Libby and Pober 2001). In addition, mast cells store proteases such as tryptase and chymase in secretory granules which can be released during graft rejection following cardiac transplantation, promoting fibrosis (Levick et al. 2011).

\section{Cellular Mediators of Cardiac Fibrosis}

The primary cellular mediator of cardiac fibrosis is the resident myocardial fibroblast. Fibroblasts are the most numerous cell type in the heart, and in response to a wide variety of stimuli, can rapidly undergo phenotype conversion to myofibroblasts (Bagchi et al. 2016b; Nagalingam et al. 2016). Fibroblasts synthesize relatively smaller amounts of ECM as well as 
MMPs in order to maintain ECM homeostasis in the otherwise healthy heart. Following conversion to myofibroblasts, ECM synthesis is dramatically increased, which overtime leads to fibrosis. Myofibroblasts not only express high levels of fibrillar collagens, but also exhibit increased expression of various markers such as fibronectin and vimentin (Bagchi et al. 2016b; Santiago et al. 2010). In addition, proteins not expressed in fibroblasts are induced, including periostin as well as $\alpha$-smooth muscle actin ( $\alpha$ SMA) which confers myofibroblast contractility (Bagchi et al. 2016b). Recently it was shown that all periostin-expressing myofibroblasts arise from Tcf21-expressing cardiac fibroblasts, and that deletion of periostin-positive cells reduces generation of the scar post-MI (Kanisicak et al. 2016). This finding is striking, since it was previously demonstrated that Tcf 21 gene deletion results in the absence of cardiac fibroblasts (Acharya et al. 2012). Thus, a Tcf21-positive lineage appears to represent at least the large majority of cardiac fibroblasts that contribute to healing after infarction.

Periostin-positive myofibroblasts appear to somewhat reverse their phenotype, becoming more like fibroblasts, following infarct healing, however it has also been reported that activated myofibroblasts may persist in the heart even decades after the initial injury, contributing to fibrosis (Kanisicak et al. 2016; Willems et al. 1994). Temporary fibroblast activation to myofibroblasts is a key event in dermal wound healing, but eventually these myofibroblasts undergo apoptosis and effectively vanish once the injury is fully healed (Czubryt 2012). It is thus tempting to speculate that the development of fibrosis in the heart results from an impairment of apoptosis, from a failure of myofibroblasts to revert to fibroblasts, or from some combination of the two phenomena.

It has been proposed that other cell populations can undergo phenotype conversion to a myofibroblast-like phenotype. For example, endothelial cells may undergo endothelial- 
mesenchymal transition (EndMT), a process that may be important in fibrotic development following pressure overload (Zeisberg et al. 2007). Similarly, epithelial-mesenchymal transition (EMT) may also contribute to scar formation (Zhou and $\mathrm{Pu} 2011$ ). Finally, it has been proposed that circulating myeloid cells may invade the myocardium, where they may stimulate endogenous cells to convert to myofibroblasts or possibly even convert to a myofibroblast-like state themselves (Haudek et al. 2006). Ultimately, the most important source of myofibroblasts may vary depending on the specific nature of the cardiac insult (Crawford et al. 2012).

Circulating fibrocytes are another potential source of collagen during the development of cardiac fibrosis (Lin et al. 2016). These multifunctional, monocyte-derived cells exhibit features of both macrophages and fibroblasts (Bucala et al. 1994). Upon cardiac insult, the local release of chemotactic factors attracts circulating fibrocytes from the bloodstream to the heart. Initially, fibrocytes are involved in the inflammatory process, expressing hematopoietic genes encoding chemokine receptors and cytokines such as CD34 and CD45, facilitating recruitment of additional inflammatory cells (Reilkoff et al. 2011). As the transition to the remodeling phase begins, fibrocytes differentiate into myofibroblast-like cells, up-regulating expression of $\alpha$-SMA, fibronectin and collagen, and down-regulating expression of CD34 and CD45 (Kao et al. 2011). This process is facilitated by TGF $\beta$ (Abe et al. 2001). Multiple experimental studies have shown that the level of circulating fibrocytes is highly elevated in the heart, and in correlation with the degree of cardiac fibrosis, in a host of cardiac pathologies including hypertension, heart failure, atrial fibrillation and even natural aging (Chu et al. 2010; Keeley et al. 2012; Szardien et al. 2012; Xie et al. 2014). However, while it is clear that fibrocytes can adopt a myofibroblast-like phenotype, it is still unclear to what degree this process actually contributes to fibrosis. 


\section{Signaling Pathways Contributing to Cardiac Fibrosis}

A wide range of paracrine signals has been shown to activate the mechanisms underlying fibrosis, including a number of growth factors and the renin-angiotensin-aldosterone system (RAAS) (Figure 2) (Leask 2010). Possibly the most potent of these signals is the cytokine TGF $\beta$, the three isoforms of which are produced by a variety of cell types including fibroblasts and those involved in the inflammatory response. Previously synthesized TGF $\beta$ can also be kept trapped within the ECM in latent complexes, to be released by mechanical forces acting upon these complexes (Buscemi et al. 2011). TGF $\beta$ plays a major role in the pathogenesis of cardiac fibrotic remodeling. Following MI, TGF $\beta$ deactivates macrophages to facilitate the switching of the infarcted heart from the inflammatory phase to scar formation. More directly, however, TGF $\beta$ also induces the phenotype conversion of fibroblasts to myofibroblasts and the transactivation of ECM component genes such as those encoding fibrillar collagens (Bujak and Frangogiannis 2007; Dobaczewski et al. 2011).

TGF $\beta$ signals via both a canonical and a non-canonical intracellular signaling pathway (Dobaczewski et al. 2011). In both pathways, extracellular TGF $\beta$ binds to and activates its type II receptor (T $\beta$ RII) dimer at the cell surface, which then recruits a type I receptor dimer that is phosphorylated by T $\beta$ RII. This event in turn activates sequential downstream intracellular cascades. In the canonical pathway, transcription factors called Smad proteins, including Smad2 and Smad3, are phosphorylated by the TGF $\beta$ receptor heterotetramer complex, resulting in their binding of Co-Smad4 and translocation to the cell nucleus where transactivation of fibrotic genes occurs (Dobaczewski et al. 2011; Zeglinski et al. 2016). In vivo and in vitro studies suggest that Smad3 signaling mediates most of the pro-fibrotic activities of TGF $\beta$ by regulating fibroblast phenotype (Dobaczewski et al. 2010; Flanders 2004). 
In the non-canonical TGF $\beta$ pathway, the signal transmitted through T $\beta$ RII activates several downstream mitogen-activated protein kinases (MAPK), including c-Jun N-terminal kinase (JNK), p38 MAPK and TGF $\beta$-activated kinase (TAK1). Each of these MAPKs phosphorylates a number of transcription factors such as c-Jun, altering their activities (Yang et al. 2003). In turn, these transcription factors regulate the expression of ECM proteins, $\alpha$ SMA and other target genes involved in cardiac fibrosis (Kompa et al. 2008; Ono et al. 2003).

The RAAS has also been shown to play a role in the pathogenesis of cardiac remodeling, including fibrosis. RAAS activation increases cardiac preload and afterload, thus increasing cardiac work and activating myocardial remodeling, including fibrosis (Dostal 2000). At least part of the effect of RAAS activation on fibrosis may stem from interactions with the TGF $\beta$ signaling pathway. TGF $\beta$ mRNA expression is up-regulated by the activation of angiotensin type 1 receptor in cardiac fibroblasts and myocytes (Rosenkranz 2004). In addition, angiotensin II regulates the expression by cardiac fibroblasts of the glycoprotein endoglin, a component of the TGF $\beta$ receptor complex, resulting in an increase in responsiveness to TGF $\beta$ (Chen et al. 2004). It is thus not surprising that the clinical use of angiotensin receptor blockade, renin inhibitors and angiotensin converting enzyme inhibitors, reduces the progression of fibrosis in heart failure (Ma et al. 2010; Zhi et al. 2013).

Connective tissue growth factor (CTGF), also known as CCN2, is a matricellular protein that has been implicated in modulating the pathological cardiac remodeling process (Daniels et al. 2009). Expression of CTGF in myocytes and fibroblasts is induced by many factors including TGF $\beta$, AngII, aldosterone, endothelin-1 and mechanical stress (Travers et al. 2016). A number of studies have shown that CTGF mRNA and protein levels are strongly up-regulated in many cardiac disease conditions (Au et al. 2011). Interestingly, these levels are elevated even before 
the induction of TGF $\beta$ expression or initiation of the fibrotic process, implicating CTGF in collagen synthesis and formation of the fibrotic scar (Duncan et al. 1999). Persistent fibrotic tissue formation may require the contribution of both TGF $\beta$ to induce fibrosis, and CTGF to maintain it (Mori et al. 1999).

PDGF has also been implicated in the pathogenesis of cardiac fibrosis by inducing TGF $\beta$ synthesis, while conversely TGF $\beta$ induces PDGF production (Czuwara-Ladykowska et al. 2001; Zhao et al. 2013). After MI, PDGF signals through PDGF receptors $\alpha$ and $\beta$ to activate overlapping signal transduction pathways, including phosphatidylinositol 3-kinase, Ras-MAPK, Src family kinases, and phospholipase $\mathrm{C} \gamma$, promoting collagen deposition, stimulating fibroblast proliferation and stabilizing the scar (Taylor 2000).

Besides growth factors, changes in physical forces applied to fibroblasts, including surrounding matrix tension and mechanical stretch, may also induce conversion to myofibroblasts and subsequent ECM synthesis. It has long been known that plating primary fibroblasts onto firm substrates, including even tissue culture plastic ware, induces rapid conversion to myofibroblasts (Hinz 2009). Physical force, acting through integrins, up-regulates multiple pro-fibrotic genes including $\alpha$-SMA and fibrillar collagens I and III (Carver et al. 1991; Roche et al. 2016). The resulting increase in ECM synthesis may represent an attempt to resist excessive extracellular forces via the construction of stronger matrix. The induction of fibroblast phenotype transition would be expected to result in increased cell adherence to the surrounding matrix, attenuating their migration and proliferation to contribute to scar maturation and stability (Tomasek et al. 2002).

Our laboratory has reported that a transcription factor called scleraxis is a central downstream effector of multiple pro-fibrotic signaling pathways in cardiac fibroblasts and 
myofibroblasts. This basic helix-loop-helix family member binds to the E-box consensus sequence CANNTG in order to transactivate target gene promoters (Cserjesi et al. 1995; Espira et al. 2009). We have found that scleraxis, while capable of inducing gene expression on its own, appears to work most potently with partners such as E12 (Espira et al. 2009). Notably, scleraxis binds to and acts synergistically with Smad3, but not Smad2, to regulate the expression of collagen I $\alpha 2$ (Bagchi and Czubryt 2012; Bagchi et al. 2016b). Furthermore, loss of scleraxis attenuates the effect of Smad3 on this target gene, and vice versa, thus scleraxis and Smad3 require one another's presence for full activity. A scleraxis mutant lacking its basic DNA binding domain acts in a dominant negative fashion to attenuate target gene expression, and in the case of collagen I $\alpha 2$, does so by recruiting Smad3 away from Smad Binding Elements, thereby impairing transcription complex formation (Bagchi et al. 2016b).

Scleraxis exerts a number of significant biological effects on cardiac fibroblasts. Besides direct transcriptional induction of collagen I $\alpha 2$ and likely I $\alpha 1$, scleraxis appears to control expression of other ECM components including fibronectin, proteoglycans and MMPs, and scleraxis gene deletion results in a loss of half of the cardiac ECM in mice (Bagchi et al. 2016a; Bagchi et al. 2016b). While scleraxis attenuates expression of $\alpha$ SMA in mesangial cells, it upregulates $\alpha \mathrm{SMA}$ in cardiac fibroblasts via direct transactivation of its gene promoter, conferring contractility (Abe et al. 2012; Bagchi et al. 2016b). Furthermore, knockdown of scleraxis completely attenuates TGF $\beta$-induced cell contraction, demonstrating an absolute requirement for scleraxis in this process. Scleraxis is thus sufficient to drive fibroblast to myofibroblast phenotype conversion: it induces expression of ECM genes and $\alpha$ SMA, confers contractility, and as we have recently shown, causes cell cycle exit and inhibition of motility (Roche et al. 2016). Over-expression of scleraxis is even capable of reprogramming epithelial cells to mesenchymal 
cells expressing fibroblast and myofibroblast marker genes, apparently via direct transcriptional regulation of EMT genes Twist1 and Snail1 (Bagchi et al. 2016b). Conversely, loss of scleraxis appears to have to opposite effect, driving cells back towards a less-pathologic fibroblast phenotype. Together, this data demonstrates that scleraxis is a pro-fibrotic regulator of cell phenotype in cardiac fibroblasts.

Scleraxis expression is induced by pro-fibrotic TGF $\beta / \mathrm{Smad} 3$ signaling, and reduced by inhibitory Smad7 (Bagchi and Czubryt 2012). TGF $\beta$ also appears to be capable of inducing scleraxis expression via a Smad-independent non-canonical pathway, possibly through c-Jun which has also been implicated in fibrosis (Zeglinski et al. 2016). Given the central roles of these pathways in fibrosis, it is thus not surprising that scleraxis expression increases significantly in the cardiac infarct scar, suggesting it may play a key causative role in the fibrotic process in vivo (Espira et al. 2009). Mechanical stretch also induces scleraxis expression, and knockdown of scleraxis attenuates the ability of stretch to cause fibroblast to myofibroblast conversion (Roche et al. 2016). However, while stretch causes transactivation of the scleraxis promoter, the precise mechanism and transcriptional regulator involved is yet to be elucidated.

\section{Current Treatments for Cardiac Fibrosis}

Despite the fact that cardiac fibrosis represents a significant risk factor for patient morbidity and mortality, at present there are no pharmacological treatments that directly target the fibrotic process. Given the vast pharmacopoeia available for treating cardiovascular disorders in general, this treatment gap is particularly conspicuous, and presents a key weakness in the management of cardiac patients. There are a number of reasons why treatment strategies for cardiac fibrosis are lacking, despite a number of efforts in this regard. Part of the problem is that, until recently, 
studies into the molecular details of how fibrosis occurs have lagged behind other areas of investigation into cardiovascular pathophysiology. Understanding the mechanisms responsible for initiation, progression and resolution of cardiac fibrosis is thus crucial to designing novel anti-fibrotic treatment strategies for patients with cardiovascular diseases.

Given the central role of TGF $\beta$ signaling in the pathogenesis of fibrosis, it is not surprising that there have been multiple attempts to interfere with this pathway. Neutralizing anti-TGF $\beta$ antibodies bind directly to the extracellular ligand to prevent receptor activation, and have been used to attenuate diastolic dysfunction and cardiac fibrosis in pressure-overloaded rats (Kuwahara et al. 2002). In contrast, administration of anti-TGF $\beta$ antibodies either before or after aortic ligation in an MI mouse model resulted in an increase in mortality and a worsening of ventricular remodeling (Frantz et al. 2008). While human clinical trials of anti-TGF $\beta$ antibodies have not yet been attempted for cardiovascular diseases, systemic antibody administration in patients with early phase systemic sclerosis was insufficient to improve disease outcome (Denton et al. 2007). The efficacy of anti-TGF $\beta$ antibodies thus remains to be established in human fibrotic diseases.

A related approach is the blockade of the TGF $\beta$ type 1 receptor using a small molecule inhibitor, which attenuated systolic dysfunction and left ventricular remodeling in an experimental rat model of MI (Tan et al. 2010). However, applying this strategy in the clinical setting is challenging, since delivery of these small molecules may impact multiple downstream pathways, raising the possibility of having off-target effects unrelated to TGF $\beta$ signaling (Varga and Pasche 2008). Indeed, the broad range effects mediated by TGF $\beta$ increases the likelihood that blockade of TGF $\beta$ have untoward off-target effects, including abrogation of matrix preserving pathways, leading to unopposed matrix degradation, cardiac dilation and dysfunction. 
Prolonged inhibition of TGF $\beta$ may also interfere with normal immune regulation and wound healing processes, as TGF $\beta$ knockout in mice results in excessive inflammation and perinatal death (Kulkarni et al. 1993).

These issues may explain the mixed results obtained following inhibition of TGF $\beta$ signaling in post-MI healing. Delivery of a TGF $\beta$ receptor II extracellular domain decoy, capable of binding TGF $\beta$, starting at 7 days prior to experimentally-induced MI in mice resulted in an increase in mortality immediately post-MI concomitant with dilatation of the left ventricle and contractile dysfunction (Ikeuchi et al. 2004). In contrast, delivery of the same decoy immediately after MI and again at 1 week resulted in improved LV function and structure, indicating the precise control over the timing of TGF $\beta$ inhibition may yield benefits while avoiding the negative effects that may occur. A subsequent study in which the decoy was delivered by adenovirus 3 days post-MI showed similar results (Okada et al. 2005).

Other issues have involved concerns with specificity of drug delivery, effective dose or offtarget effects. For example, given the key role of inflammation in the pathogenesis of fibrosis, targeting of the inflammatory process after cardiac injury could potentially be beneficial. However, as noted above, inflammation is a key phase of the healing process post-MI, thus antiinflammatory strategies may be problematic in this particular scenario. Furthermore, systemic delivery of anti-inflammatories through injection or oral intake may result in an insufficient dose for efficacy in treating the diseased myocardium (Fan and Guan 2016; Rosenbloom et al. 2013). While targeting TGF $\beta$ signaling has promise, caution is required since TGF $\beta$ has an antiproliferative effect in some cell types, thus attenuation of TGF $\beta$ signaling could promote oncogenesis unintentionally in other tissues (Connolly et al. 2012). Targeting of other profibrotic pleiotropic cytokines and growth factors, such as angiotensin II, CTGF and PDGF that 
are directly and indirectly involved in myofibroblast differentiation, may suffer from similar offtarget effects (Leask 2010).

At present, while direct inhibition of fibrosis mechanisms has remained elusive, modest success has instead come from treating the underlying cardiovascular pathologies that initiate or facilitate the development of fibrosis, or from delivering cardioprotective drugs to reduce stress and damage to the heart. In some cases, these approaches appear to work via secondary mechanisms that are not yet understood. Anti-hypertensive drugs, for example, have shown antifibrotic effects in the myocardium by attenuating remodeling secondary to hypertensive heart disease. Calcium channel blockers, beta-blockers and RAAS inhibitors, including angiotensin converting enzyme inhibitors, angiotensin II receptor blockers and aldosterone antagonists, exhibit beneficial effects on cardiac remodeling independently of their impact on blood pressure, with demonstrated improvements in left ventricular mass regression and diastolic function (Cuspidi et al. 2008; Diamond and Phillips 2004). Similarly, combination therapy of angiotensin II receptor blockers with angiotensin converting enzyme inhibitors may attenuate cardiac remodeling and improve patient prognosis after myocardial infarction (Leask 2010; Nakamura et al. 2003). Statins are used primarily to lower serum cholesterol through inhibition of 3-hydroxy3-methylglutaryl-coenzyme A reductase, but have shown surprising effects in the inhibition of scarring and cardiac remodeling via an anti-fibrotic activity (Gourdie et al. 2016).

Surgical resection of scar tissue is another strategy that has been used therapeutically - not to treat fibrosis itself, but instead to reduce arrhythmias arising from the electrical heterogeneity introduced by scar tissue. Encircling endocardial ventriculotomy was used to treat patients with ventricular tachycardia post-MI, and who were resistant to medical treatment. By excluding the 
damaged region of the myocardium, this approach successfully eliminated tachyarrhythmias in a small number of patients up to 24 months after surgery (Guiraudon et al. 1978).

\section{Novel Approaches to Treating Cardiac Fibrosis}

The quest for new therapies that specifically target the underlying mechanisms of fibrosis continues, and in fact is accelerating as the critical role played by fibrosis in tissue dysfunction throughout the body becomes more apparent. Large numbers of potential inhibitors of fibrosis are currently under study, although in many cases the mechanism of action is unknown (Nanthakumar et al. 2015). One small molecular inhibitor that has passed beyond clinical trials to human use is pirfenidone.

A number of studies have investigated the therapeutic effect of pirfenidone on fibrotic disease in various animal models and multiple organs, including the lungs, kidneys, liver and heart (Schaefer et al. 2011). Following extensive clinical trials, pirfenidone was approved for clinical use in idiopathic pulmonary fibrosis (IPF) under the trade name Esbriet (Bando 2016).It appears capable of slowing or inhibiting the progression of fibrotic lesions, and prevents the formation of new lesions following injury, however it is noteworthy that its mechanism of action is still not fully understood. While clearly not a cure for IPF, pirfenidone has nonetheless demonstrated clinically significant improvements in lung function and survival of patients with mild to moderate IPF both in clinical trials and in real-world usage (Noble et al. 2016). It has not yet been widely approved for patient use in other tissues, although liver fibrosis and chronic dermal wounds are promising targets.

Pirfenidone alters expression of type I collagen, as well as of cytokines including TGF $\beta$, TNF $\alpha$, and PDGF, attenuating fibrosis in various chronic diseases, although exactly how it exerts 
these effects is unclear (Lopez-de la Mora et al. 2015). In vitro studies have demonstrated that pirfenidone, when applied to isolated cardiac fibroblasts, reduces the synthesis and secretion of TGF $\beta$, modulates fibroblast proliferation and attenuates $\alpha$ SMA expression and cell contractility (Shi et al. 2011). Pirfenidone attenuated cardiac remodeling in hypertensive rats independently of blood pressure, in a post-MI rat model reduced scar size, improved left ventricular function and decreased the incidence of arrhythmias (Mirkovic et al. 2002; Nguyen et al. 2010). Pirfenidone thus shows promise for the modulation of cardiac fibrosis in human disease.

Pirfenidone can also act as an immune modulator, and has been used in allograft rejection to minimize the amount of scarring following surgery (Visner et al. 2009). To this end, pirfenidone has been shown to suppress inflammasome formation in cardiac remodeling induced by pressure overload (Roche and Czubryt 2013; Wang et al. 2013). Thus it is possible that pirfenidone exerts its action, not directly on the intracellular mechanism of fibrosis, but rather on inflammation that activates the fibrotic process upstream of these pathways. Supporting data for this model comes from a study in which oral pirfenidone was administered to mdx Duchenne muscular dystrophy mice, resulting in an improvement in cardiac contractility but without a change in cardiac stiffness or in the amount of fibrosis, suggesting that the drug's mechanism of action is not on fibrotic intracellular signaling pathways (Van Erp et al. 2006).

The only other drug currently approved specifically for treating a fibrotic disease in humans is nintedanib, which, like pirfenidone, has been approved for IPF, and which is also undergoing clinical trials for systemic sclerosis (Fala 2015). Unlike pirfenidone, there is a better understanding of the mechanism of action of nintedanib, which blocks various receptor tyrosine kinases implicated in fibrosis including those of PDGF, fibroblast growth factor and vascular endothelial growth factor (Nanthakumar et al. 2015). This drug has shown significant anti- 
fibrotic and anti-inflammatory properties in animal models of IPF (Wollin et al. 2014). Despite the promising nature of nintedanib, it has not yet been tested in animal models of cardiac fibrosis.

The central role for scleraxis in mediating fibroblast and myofibroblast phenotype makes it an intriguing target for the development of anti-fibrotic therapies. In our various studies, any interference with scleraxis function - whether by gene deletion, knockdown, use of a dominant negative, or alteration of post-translational modifications - attenuated evidence of fibroblast to myofibroblast transition and reduced expression of collagen, other ECM components or myofibroblast markers (Bagchi and Czubryt 2012; Bagchi et al. 2016a; Bagchi et al. 2016b; Bagchi et al. 2016c; Espira et al. 2009; Roche et al. 2016). This effect occurred regardless of whether the upstream inducer of fibrosis was TGF $\beta$, Smad3 or mechanical stretch, suggesting that scleraxis acts as a signaling nexus upon which multiple pro-fibrotic pathways descend. Drugging scleraxis may thus have the dual benefits of reducing off-target effects and increasing efficacy by blocking the common effector of multiple fibrotic signaling pathways, rather than by targeting upstream inducers of these pathways such as cytokines or their receptors. Furthermore, if scleraxis is involved in fibrotic diseases of other tissues such as the lung or kidneys, the creation of anti-scleraxis therapies may provide the added benefit of having a single treatment for fibrosis regardless of the tissue type involved.

There are several strategies that could be developed to target scleraxis. One approach is knockdown via adenovirus-mediated gene delivery of a suitable, specific shRNA. We have successfully employed this approach in vitro to nearly eliminate scleraxis levels and attenuate scleraxis-dependent ECM gene expression (Bagchi et al. 2016a; Bagchi et al. 2016b; Roche et al. 2016). A potential refinement for this approach would be to drive the expression of the shRNA 
with a cell type-specific gene promoter, such as that for TCF21, which would facilitate delivery to cardiac fibroblasts but not to other cells of the myocardium, thus reducing off-target effects (Acharya et al. 2012). This approach would also permit tuning of the degree of knockdown by controlling viral load, as well as of the length of treatment. The use of drug-inducible promoters could permit further refinement.

Alternatively, it may be possible to alter scleraxis activity, rather than expression, via the development of small molecule inhibitors. Our work has shown that scleraxis requires constitutive phosphorylation for its transactivation activity, and that scleraxis activity further relies on its ability to bind to protein partners and DNA (Bagchi and Czubryt 2012; Bagchi et al. 2016b; Bagchi et al. 2016c; Espira et al. 2009). These requirements open the door to novel ways to interfere with scleraxis function. Other laboratories have demonstrated the attenuation of the activity of transcription factors such as NOTCH via the use of specific inhibitors such as stapled peptides - an approach that may be similarly useful for scleraxis (Moellering et al. 2009).

\section{Conclusion}

Cardiac fibrosis is a common pathology that is both a response to stress and damage, and a cause of further dysfunction leading to heart failure. Its very ubiquity, coupled with its significant negative impact on patient outcomes, requires a concerted effort to develop novel treatments to either arrest the progress of fibrosis, or even to reverse it. New treatments like pirfenidone, and new targets like scleraxis, provide tantalizing approaches that may be capable of achieving these therapeutic goals. Further research in this field is urgently needed, and has tremendous potential to exert powerful positive effects on the health and survival of patients with fibrotic diseases. 
https://mc06.manuscriptcentral.com/cjpp-pubs 


\section{References}

Abe, H., Tominaga, T., Matsubara, T., Abe, N., Kishi, S., Nagai, K., et al. 2012. Scleraxis modulates bone morphogenetic protein 4 (BMP4)-Smad1 protein-smooth muscle alpha-actin (SMA) signal transduction in diabetic nephropathy. J. Biol. Chem. 287(24): 20430-20442.

Abe, R., Donnelly, S. C., Peng, T., Bucala, R., and Metz, C. N. 2001. Peripheral blood fibrocytes: differentiation pathway and migration to wound sites. J. Immunol. 166(12): 7556-7562.

Acharya, A., Baek, S. T., Huang, G., Eskiocak, B., Goetsch, S., Sung, C. Y., et al. 2012. The bHLH transcription factor Tef21 is required for lineage-specific EMT of cardiac fibroblast progenitors. Development, 139(12): 2139-2149.

Almehmadi, F., Joncas, S. X., Nevis, I., Zahrani, M., Bokhari, M., Stirrat, J., et al. 2014. Prevalence of myocardial fibrosis patterns in patients with systolic dysfunction: prognostic significance for the prediction of sudden cardiac arrest or appropriate implantable cardiac defibrillator therapy. Circ. Cardiovasc. Imaging, 7(4): 593-600.

Assomull, R. G., Prasad, S. K., Lyne, J., Smith, G., Burman, E. D., Khan, M., et al. 2006. Cardiovascular magnetic resonance, fibrosis, and prognosis in dilated cardiomyopathy. J. Am. Coll. Cardiol. 48(10): 1977-1985.

Au, C. G., Butler, T. L., Sherwood, M. C., Egan, J. R., North, K. N., and Winlaw, D. S. 2011. Increased connective tissue growth factor associated with cardiac fibrosis in the mdx mouse model of dystrophic cardiomyopathy. Int. J. Exp. Pathol. 92(1): 57-65.

Bagchi, R. A., and Czubryt, M. P. 2012. Synergistic roles of scleraxis and Smads in the regulation of collagen 1alpha2 gene expression. Biochim. Biophys. Acta, 1823(10): 1936-1944.

Bagchi, R. A., Lin, J., Wang, R., and Czubryt, M. P. 2016a. Regulation of fibronectin gene expression in cardiac fibroblasts by scleraxis. Cell Tissue Res. 366(2): 381-391.

Bagchi, R. A., Roche, P., Aroutiounova, N., Espira, L., Abrenica, B., Schweitzer, R., et al. 2016b. The transcription factor scleraxis is a critical regulator of cardiac fibroblast phenotype. BMC Biol. 14(1): 21. 
Bagchi, R. A., Wang, R., Jahan, F., Wigle, J. T., and Czubryt, M. P. 2016c. Regulation of scleraxis transcriptional activity by serine phosphorylation. J. Mol. Cell. Cardiol. 92: 140-148.

Bando, M. 2016. Pirfenidone: Clinical trials and clinical practice in patients with idiopathic pulmonary fibrosis. Respir. Investig. 54(5): 298-304.

Batlle, M., Perez-Villa, F., Lazaro, A., Garcia-Pras, E., Ramirez, J., Ortiz, J., et al. 2007. Correlation between mast cell density and myocardial fibrosis in congestive heart failure patients. Transplant. Proc. 39(7): 2347-2349.

Bonne, G., Carrier, L., Richard, P., Hainque, B., and Schwartz, K. 1998. Familial hypertrophic cardiomyopathy: from mutations to functional defects. Circ. Res. 83(6): 580-593.

Bucala, R., Spiegel, L. A., Chesney, J., Hogan, M., and Cerami, A. 1994. Circulating fibrocytes define a new leukocyte subpopulation that mediates tissue repair. Mol. Med. 1(1): 71-81.

Bujak, M., and Frangogiannis, N. G. 2007. The role of TGF-beta signaling in myocardial infarction and cardiac remodeling. Cardiovasc. Res. 74(2): 184-195.

Buscemi, L., Ramonet, D., Klingberg, F., Formey, A., Smith-Clerc, J., Meister, J. J., et al. 2011. The single-molecule mechanics of the latent TGF-betal complex. Curr. Biol. 21(24): 2046-2054.

Carver, W., Nagpal, M. L., Nachtigal, M., Borg, T. K., and Terracio, L. 1991. Collagen expression in mechanically stimulated cardiac fibroblasts. Circ. Res. 69(1): 116-122.

Chen, K., Mehta, J. L., Li, D., Joseph, L., and Joseph, J. 2004. Transforming growth factor beta receptor endoglin is expressed in cardiac fibroblasts and modulates profibrogenic actions of angiotensin II. Circ. Res. 95(12): 1167-1173.

Chu, P. Y., Mariani, J., Finch, S., McMullen, J. R., Sadoshima, J., Marshall, T., et al. 2010. Bone marrow-derived cells contribute to fibrosis in the chronically failing heart. Am. J. Pathol. 176(4): 17351742.

Connolly, E. C., Freimuth, J., and Akhurst, R. J. 2012. Complexities of TGF-beta targeted cancer therapy. Int. J. Biol. Sci. 8(7): 964-978. 
Crawford, J. R., Haudek, S. B., Cieslik, K. A., Trial, J., and Entman, M. L. 2012. Origin of Developmental Precursors Dictates the Pathophysiologic Role of Cardiac Fibroblasts. J. Cardiovasc. Transl. Res. 5(6): 749-759.

Cserjesi, P., Brown, D., Ligon, K. L., Lyons, G. E., Copeland, N. G., Gilbert, D. J., et al. 1995. Scleraxis: a basic helix-loop-helix protein that prefigures skeletal formation during mouse embryogenesis. Development, 121(4): 1099-1110.

Cuspidi, C., Negri, F., and Zanchetti, A. 2008. Angiotensin II receptor blockers and cardiovascular protection: focus on left ventricular hypertrophy regression and atrial fibrillation prevention. Vasc. Health Risk Manage. 4(1): 67-73.

Czubryt, M. P. 2012. Common threads in cardiac fibrosis, infarct scar formation, and wound healing. Fibrogenesis Tissue Repair, 5(1): 19.

Czuwara-Ladykowska, J., Gore, E. A., Shegogue, D. A., Smith, E. A., and Trojanowska, M. 2001. Differential regulation of transforming growth factor-beta receptors type I and II by platelet-derived growth factor in human dermal fibroblasts. Br. J. Dermatol. 145(4): 569-575.

Daniels, A., van Bilsen, M., Goldschmeding, R., van der Vusse, G. J., and van Nieuwenhoven, F. A. 2009. Connective tissue growth factor and cardiac fibrosis. Acta Physiol. (Oxf.) 195(3): 321-338. de Jong, S., van Veen, T. A., van Rijen, H. V., and de Bakker, J. M. 2011. Fibrosis and cardiac arrhythmias. J. Cardiovasc. Pharmacol. 57(6): 630-638.

Debonnaire, P., Delgado, V., and Bax, J. J. 2015. Potential role of fibrosis imaging in severe valvular heart disease. Heart, 101(5): 397-407.

Denton, C. P., Merkel, P. A., Furst, D. E., Khanna, D., Emery, P., Hsu, V. M., et al. 2007. Recombinant human anti-transforming growth factor beta1 antibody therapy in systemic sclerosis: a multicenter, randomized, placebo-controlled phase I/II trial of CAT-192. Arthritis Rheum. 56(1): 323-333. Diamond, J. A., and Phillips, R. A. 2004. Antihypertensive drugs and the heart. Curr. Cardiol. Rep. 6(6): 409-415. 
Dobaczewski, M., Bujak, M., Li, N., Gonzalez-Quesada, C., Mendoza, L. H., Wang, X. F., et al. 2010. Smad3 signaling critically regulates fibroblast phenotype and function in healing myocardial infarction. Circ. Res. 107(3): 418-428.

Dobaczewski, M., Chen, W., and Frangogiannis, N. G. 2011. Transforming growth factor (TGF)-beta signaling in cardiac remodeling. J. Mol. Cell. Cardiol. 51(4): 600-606.

Dostal, D. E. 2000. The cardiac renin-angiotensin system: novel signaling mechanisms related to cardiac growth and function. Regul. Pept. 91(1-3): 1-11.

Duncan, M. R., Frazier, K. S., Abramson, S., Williams, S., Klapper, H., Huang, X., et al. 1999.

Connective tissue growth factor mediates transforming growth factor beta-induced collagen synthesis: down-regulation by cAMP. FASEB J. 13(13): 1774-1786.

Espira, L., Lamoureux, L., Jones, S. C., Gerard, R. D., Dixon, I. M., and Czubryt, M. P. 2009. The basic helix-loop-helix transcription factor scleraxis regulates fibroblast collagen synthesis. J. Mol. Cell. Cardiol. 47(2): 188-195.

Fala, L. 2015. Ofev (Nintedanib): First Tyrosine Kinase Inhibitor Approved for the Treatment of Patients with Idiopathic Pulmonary Fibrosis. Am. Health Drug Benefits, 8(Spec Feature): 101-104.

Fan, Z., and Guan, J. 2016. Antifibrotic therapies to control cardiac fibrosis. Biomater. Res. 20: 13. Flanders, K. C. 2004. Smad3 as a mediator of the fibrotic response. Int. J. Exp. Pathol. 85(2): 47-64. Frantz, S., Hu, K., Adamek, A., Wolf, J., Sallam, A., Maier, S. K., et al. 2008. Transforming growth factor beta inhibition increases mortality and left ventricular dilatation after myocardial infarction. Basic. Res. Cardiol. 103(5): 485-492.

Gourdie, R. G., Dimmeler, S., and Kohl, P. 2016. Novel therapeutic strategies targeting fibroblasts and fibrosis in heart disease. Nat. Rev. Drug Discov. 15(9): 620-638.

Guiraudon, G., Fontaine, G., Frank, R., Escande, G., Etievent, P., and Cabrol, C. 1978. Encircling endocardial ventriculotomy: a new surgical treatment for life-threatening ventricular tachycardias resistant to medical treatment following myocardial infarction. Ann. Thorac. Surg. 26(5): 438-444. 
Hara, M., Ono, K., Hwang, M. W., Iwasaki, A., Okada, M., Nakatani, K., et al. 2002. Evidence for a role of mast cells in the evolution to congestive heart failure. J. Exp. Med. 195(3): 375-381.

Haudek, S. B., Xia, Y., Huebener, P., Lee, J. M., Carlson, S., Crawford, J. R., et al. 2006. Bone marrowderived fibroblast precursors mediate ischemic cardiomyopathy in mice. Proc. Natl. Acad. Sci. U. S. A. 103(48): 18284-18289.

Hinz, B. 2009. Tissue stiffness, latent TGF-betal activation, and mechanical signal transduction: implications for the pathogenesis and treatment of fibrosis. Curr. Rheumatol. Rep. 11(2): 120-126.

Hor, K. N., Taylor, M. D., Al-Khalidi, H. R., Cripe, L. H., Raman, S. V., Jefferies, J. L., et al. 2013. Prevalence and distribution of late gadolinium enhancement in a large population of patients with Duchenne muscular dystrophy: effect of age and left ventricular systolic function. J. Cardiovasc. Magn. Reson. 15: 107.

Ikeuchi, M., Tsutsui, H., Shiomi, T., Matsusaka, H., Matsushima, S., Wen, J., et al. 2004. Inhibition of TGF-beta signaling exacerbates early cardiac dysfunction but prevents late remodeling after infarction. Cardiovasc. Res. 64(3): 526-535.

Kanisicak, O., Khalil, H., Ivey, M. J., Karch, J., Maliken, B. D., Correll, R. N., et al. 2016. Genetic lineage tracing defines myofibroblast origin and function in the injured heart. Nat. Commun. 7: 12260 . Kao, H. K., Chen, B., Murphy, G. F., Li, Q., Orgill, D. P., and Guo, L. 2011. Peripheral blood fibrocytes: enhancement of wound healing by cell proliferation, re-epithelialization, contraction, and angiogenesis. Ann. Surg. 254(6): 1066-1074.

Keeley, E. C., Mehrad, B., Janardhanan, R., Salerno, M., Hunter, J. R., Burdick, M. M., et al. 2012. Elevated circulating fibrocyte levels in patients with hypertensive heart disease. J. Hypertens. 30(9): $1856-1861$.

Kompa, A. R., See, F., Lewis, D. A., Adrahtas, A., Cantwell, D. M., Wang, B. H., et al. 2008. Long-term but not short-term p38 mitogen-activated protein kinase inhibition improves cardiac function and reduces cardiac remodeling post-myocardial infarction. J. Pharmacol. Exp. Ther. 325(3): 741-750. 
Kong, P., Christia, P., and Frangogiannis, N. G. 2014. The pathogenesis of cardiac fibrosis. Cell. Mol. Life Sci. 71(4): 549-574.

Kulkarni, A. B., Huh, C. G., Becker, D., Geiser, A., Lyght, M., Flanders, K. C., et al. 1993. Transforming growth factor beta 1 null mutation in mice causes excessive inflammatory response and early death. Proc. Natl. Acad. Sci. U. S. A. 90(2): 770-774.

Kuwahara, F., Kai, H., Tokuda, K., Kai, M., Takeshita, A., Egashira, K., et al. 2002. Transforming growth factor-beta function blocking prevents myocardial fibrosis and diastolic dysfunction in pressureoverloaded rats. Circulation, 106(1): 130-135.

Leask, A. 2010. Potential therapeutic targets for cardiac fibrosis: TGFbeta, angiotensin, endothelin, CCN2, and PDGF, partners in fibroblast activation. Circ. Res. 106(11): 1675-1680.

Levick, S. P., Melendez, G. C., Plante, E., McLarty, J. L., Brower, G. L., and Janicki, J. S. 2011. Cardiac mast cells: the centrepiece in adverse myocardial remodelling. Cardiovasc. Res. 89(1): 12-19. Leyva, F., Taylor, R. J., Foley, P. W., Umar, F., Mulligan, L. J., Patel, K., et al. 2012. Left ventricular midwall fibrosis as a predictor of mortality and morbidity after cardiac resynchronization therapy in patients with nonischemic cardiomyopathy. J. Am. Coll. Cardiol. 60(17): 1659-1667.

Libby, P., and Pober, J. S. 2001. Chronic rejection. Immunity, 14(4): 387-397.

Lin, R. J., Su, Z. Z., Liang, S. M., Chen, Y. Y., Shu, X. R., Nie, R. Q., et al. 2016. Role of circulating fibrocytes in cardiac fibrosis. Chin. Med. J. 129(3): 326-331.

Lopez-de la Mora, D. A., Sanchez-Roque, C., Montoya-Buelna, M., Sanchez-Enriquez, S., LucanoLanderos, S., Macias-Barragan, J., et al. 2015. Role and New Insights of Pirfenidone in Fibrotic Diseases. Int. J. Med. Sci. 12(11): 840-847.

Ma, T. K., Kam, K. K., Yan, B. P., and Lam, Y. Y. 2010. Renin-angiotensin-aldosterone system blockade for cardiovascular diseases: current status. Br. J. Pharmacol. 160(6): 1273-1292.

Mirkovic, S., Seymour, A. M., Fenning, A., Strachan, A., Margolin, S. B., Taylor, S. M., et al. 2002. Attenuation of cardiac fibrosis by pirfenidone and amiloride in DOCA-salt hypertensive rats. Br. J. Pharmacol. 135(4): 961-968. 
Moellering, R. E., Cornejo, M., Davis, T. N., Del Bianco, C., Aster, J. C., Blacklow, S. C., et al. 2009. Direct inhibition of the NOTCH transcription factor complex. Nature, 462(7270): 182-188.

Mori, T., Kawara, S., Shinozaki, M., Hayashi, N., Kakinuma, T., Igarashi, A., et al. 1999. Role and interaction of connective tissue growth factor with transforming growth factor-beta in persistent fibrosis: A mouse fibrosis model. J. Cell. Physiol. 181(1): 153-159.

Nagalingam, R. S., Safi, H. A., and Czubryt, M. P. 2016. Gaining myocytes or losing fibroblasts: Challenges in cardiac fibroblast reprogramming for infarct repair. J. Mol. Cell. Cardiol. 93: 108-114. Nakamura, Y., Yoshiyama, M., Omura, T., Yoshida, K., Izumi, Y., Takeuchi, K., et al. 2003. Beneficial effects of combination of ACE inhibitor and angiotensin II type 1 receptor blocker on cardiac remodeling in rat myocardial infarction. Cardiovasc. Res. 57(1): 48-54.

Nanthakumar, C. B., Hatley, R. J., Lemma, S., Gauldie, J., Marshall, R. P., and Macdonald, S. J. 2015. Dissecting fibrosis: therapeutic insights from the small-molecule toolbox. Nat. Rev. Drug. Discov. 14(10): 693-720.

Nguyen, D. T., Ding, C., Wilson, E., Marcus, G. M., and Olgin, J. E. 2010. Pirfenidone mitigates left ventricular fibrosis and dysfunction after myocardial infarction and reduces arrhythmias. Heart Rhythm, 7(10): 1438-1445.

Noble, P. W., Albera, C., Bradford, W. Z., Costabel, U., du Bois, R. M., Fagan, E. A., et al. 2016. Pirfenidone for idiopathic pulmonary fibrosis: analysis of pooled data from three multinational phase 3 trials. Eur. Respir. J. 47(1): 243-253.

Okada, H., Takemura, G., Kosai, K., Li, Y., Takahashi, T., Esaki, M., et al. 2005. Postinfarction gene therapy against transforming growth factor-beta signal modulates infarct tissue dynamics and attenuates left ventricular remodeling and heart failure. Circulation, 111(19): 2430-2437.

Ono, K., Ohtomo, T., Ninomiya-Tsuji, J., and Tsuchiya, M. 2003. A dominant negative TAK1 inhibits cellular fibrotic responses induced by TGF-beta. Biochem. Biophys. Res. Commun. 307(2): 332-337. Pichler, M., Rainer, P. P., Schauer, S., and Hoefler, G. 2012. Cardiac fibrosis in human transplanted hearts is mainly driven by cells of intracardiac origin. J. Am. Coll. Cardiol. 59(11): 1008-1016. 
Prabhu, S. D., and Frangogiannis, N. G. 2016. The biological basis for cardiac repair after myocardial infarction: from inflammation to fibrosis. Circ. Res. 119(1): 91-112.

Querejeta, R., Lopez, B., Gonzalez, A., Sanchez, E., Larman, M., Martinez Ubago, J. L., et al. 2004. Increased collagen type I synthesis in patients with heart failure of hypertensive origin: relation to myocardial fibrosis. Circulation, 110(10): 1263-1268.

Reilkoff, R. A., Bucala, R., and Herzog, E. L. 2011. Fibrocytes: emerging effector cells in chronic inflammation. Nat. Rev. Immunol. 11(6): 427-435.

Roche, P., and Czubryt, M. P. 2013. Pirfenidone and the inflammasome: getting to the heart of cardiac remodeling. Cardiology, 126(1): 59-61.

Roche, P. L., Filomeno, K. L., Bagchi, R. A., and Czubryt, M. P. 2015. Intracellular signaling of cardiac fibroblasts. Compr. Physiol. 5(2): 721-760.

Roche, P. L., Nagalingam, R. S., Bagchi, R. A., Aroutiounova, N., Belisle, B. M., Wigle, J. T., et al. 2016. Role of scleraxis in mechanical stretch-mediated regulation of cardiac myofibroblast phenotype. Am. J. Physiol. Cell Physiol. 311(2): C297-307.

Rosenbloom, J., Mendoza, F. A., and Jimenez, S. A. 2013. Strategies for anti-fibrotic therapies. Biochim. Biophys. Acta 1832(7): 1088-1103.

Rosenkranz, S. 2004. TGF-beta1 and angiotensin networking in cardiac remodeling. Cardiovasc. Res. 63(3): 423-432.

Santiago, J. J., Dangerfield, A. L., Rattan, S. G., Bathe, K. L., Cunnington, R. H., Raizman, J. E., et al. 2010. Cardiac fibroblast to myofibroblast differentiation in vivo and in vitro: expression of focal adhesion components in neonatal and adult rat ventricular myofibroblasts. Dev. Dyn. 239(6): 1573-1584.

Schaefer, C. J., Ruhrmund, D. W., Pan, L., Seiwert, S. D., and Kossen, K. 2011. Antifibrotic activities of pirfenidone in animal models. Eur. Respir. Rev. 20(120): 85-97.

Schiechl, G., Hermann, F. J., Rodriguez Gomez, M., Kutzi, S., Schmidbauer, K., Talke, Y., et al. 2016. Basophils trigger fibroblast activation in cardiac allograft fibrosis development. Am. J. Transplant. 16(9): $2574-2588$. 
Shi, Q., Liu, X., Bai, Y., Cui, C., Li, J., Li, Y., et al. 2011. In vitro effects of pirfenidone on cardiac fibroblasts: proliferation, myofibroblast differentiation, migration and cytokine secretion. PLoS One, 6(11): e28134.

Szardien, S., Nef, H. M., Troidl, C., Willmer, M., Voss, S., Liebetrau, C., et al. 2012. Bone marrowderived cells contribute to cell turnover in aging murine hearts. Int. J. Mol. Med. 30(2): 283-287.

Talman, V., and Ruskoaho, H. 2016. Cardiac fibrosis in myocardial infarction-from repair and remodeling to regeneration. Cell Tissue Res. 365(3): 563-581.

Tan, S. M., Zhang, Y., Connelly, K. A., Gilbert, R. E., and Kelly, D. J. 2010. Targeted inhibition of activin receptor-like kinase 5 signaling attenuates cardiac dysfunction following myocardial infarction. Am. J. Physiol. Heart. Circ. Physiol. 298(5): H1415-1425.

Tarone, G., Balligand, J. L., Bauersachs, J., Clerk, A., De Windt, L., Heymans, S., et al. 2014. Targeting myocardial remodelling to develop novel therapies for heart failure: a position paper from the Working Group on Myocardial Function of the European Society of Cardiology. Eur. J. Heart Fail. 16(5): 494-508. Taylor, C. C. 2000. Platelet-derived growth factor activates porcine thecal cell phosphatidylinositol-3kinase-Akt/PKB and ras-extracellular signal-regulated kinase-1/2 kinase signaling pathways via the platelet-derived growth factor-beta receptor. Endocrinology, 141(4): 1545-1553. Tomasek, J. J., Gabbiani, G., Hinz, B., Chaponnier, C., and Brown, R. A. 2002. Myofibroblasts and mechano-regulation of connective tissue remodelling. Nat. Rev. Mol. Cell. Biol. 3(5): 349-363. Travers, J. G., Kamal, F. A., Robbins, J., Yutzey, K. E., and Blaxall, B. C. 2016. Cardiac fibrosis: the fibroblast awakens. Circ. Res. 118(6): 1021-1040.

Van Erp, C., Irwin, N. G., and Hoey, A. J. 2006. Long-term administration of pirfenidone improves cardiac function in mdx mice. Muscle Nerve, 34(3): 327-334.

Varga, J., and Pasche, B. 2008. Antitransforming growth factor-beta therapy in fibrosis: recent progress and implications for systemic sclerosis. Curr. Opin. Rheumatol. 20(6): 720-728. 
Visner, G. A., Liu, F., Bizargity, P., Liu, H., Liu, K., Yang, J., et al. 2009. Pirfenidone inhibits T-cell activation, proliferation, cytokine and chemokine production, and host alloresponses. Transplantation, 88(3): 330-338.

Wang, Y., Wu, Y., Chen, J., Zhao, S., and Li, H. 2013. Pirfenidone attenuates cardiac fibrosis in a mouse model of TAC-induced left ventricular remodeling by suppressing NLRP3 inflammasome formation. Cardiology, 126(1): 1-11.

Wei, L. 2011. Immunological aspect of cardiac remodeling: T lymphocyte subsets in inflammationmediated cardiac fibrosis. Exp. Mol. Pathol. 90(1): 74-78.

Willems, I. E., Havenith, M. G., De Mey, J. G., and Daemen, M. J. 1994. The alpha-smooth muscle actinpositive cells in healing human myocardial scars. Am. J. Pathol. 145(4): 868-875.

Wollin, L., Maillet, I., Quesniaux, V., Holweg, A., and Ryffel, B. 2014. Antifibrotic and antiinflammatory activity of the tyrosine kinase inhibitor nintedanib in experimental models of lung fibrosis. J. Pharmacol. Exp. Ther. 349(2): 209-220.

Wynn, T. A. 2004. Fibrotic disease and the T(H)1/T(H)2 paradigm. Nature reviews. Immunology, 4(8): $583-594$.

Wynn, T. A. 2008. Cellular and molecular mechanisms of fibrosis. J. Pathol. 214(2): 199-210.

Xie, X., Liu, Y., Gao, S., Wu, B., Hu, X., and Chen, J. 2014. Possible involvement of fibrocytes in atrial fibrosis in patients with chronic atrial fibrillation. Circ. J. 78(2): 338-344.

Yang, S. H., Sharrocks, A. D., and Whitmarsh, A. J. 2003. Transcriptional regulation by the MAP kinase signaling cascades. Gene, 320: 3-21.

Zeglinski, M. R., Roche, P., Hnatowich, M., Jassal, D. S., Wigle, J. T., Czubryt, M. P., et al. 2016. TGFbeta1 regulates Scleraxis expression in primary cardiac myofibroblasts by a Smad-independent mechanism. Am. J. Physiol. Heart Circ. Physiol. 310(2): H239-249.

Zeisberg, E. M., Tarnavski, O., Zeisberg, M., Dorfman, A. L., McMullen, J. R., Gustafsson, E., et al. 2007. Endothelial-to-mesenchymal transition contributes to cardiac fibrosis. Nat. Med. 13(8): 952-961. 
Zhao, T., Zhao, W., Chen, Y., Li, V. S., Meng, W., and Sun, Y. 2013. Platelet-derived growth factor-D promotes fibrogenesis of cardiac fibroblasts. Am. J. Physiol. Heart Circ. Physiol. 304(12): H1719-1726. Zhi, H., Luptak, I., Alreja, G., Shi, J., Guan, J., Metes-Kosik, N., et al. 2013. Effects of direct Renin inhibition on myocardial fibrosis and cardiac fibroblast function. PLoS One, 8(12): e81612.

Zhou, B., and Pu, W. T. 2011. Epicardial epithelial-to-mesenchymal transition in injured heart. J. Cell. Mol. Med. 15(12): 2781-2783. 
Figure 1: Induction of cardiac fibrosis and its downstream effects. Multiple initiating events lead to cardiac stress and damage (white ovals), which in turn directly or indirectly promote cardiac fibrosis. Over time, fibrosis adversely affects cardiac function by promoting arrhythmogenesis or systolic and diastolic dysfunction (dark gray ovals), ultimately leading to significant pathological outcomes including heart failure and sudden cardiac death (black boxes). Some initiating events, such as diabetes and aging, promote fibrosis through mechanisms that remain poorly described (dashed arrows).

Figure 2: Pro-fibrotic intracellular signaling pathways in the cardiac fibroblast. A host of cytokines have been identified as key inducers of cardiac fibrosis. The TGF $\beta$ signaling pathway (light gray box) is the primary driver of fibrosis, resulting in increased ECM gene expression and fibroblast to myofibroblast phenotype conversion. TGF $\beta$ signals via its receptor complex through two mechanisms: the canonical pathway, in which receptor-linked Smad (R-Smad) transcription factors such as Smad3 work with co-Smad4 to directly transactivate target genes; and the noncanonical pathway in which MAP kinases including JNK, p38 and TAK1 are activated to effect down-stream pro-fibrotic activity. CCN2/CTGF, scleraxis (Scx) and PDGF are induced by TGF $\beta$ signaling as well as other pro-fibrotic pathways including angiotensin II (AngII), endothelin-1 (ET1), aldosterone (Aldo), and mechanical stretch; in turn, these factors feed forward to further promote fibrosis. For example, scleraxis directly transactivates ECM target genes, can also bind to and synergize with Smad3 to control ECM gene expression, and is critically required for the function of TGF $\beta$ signaling. The activation of pro-fibrotic signaling by a host of up-stream inducers thus causes a powerful, self-reinforcing response leading to tissue fibrosis and dysfunction. 


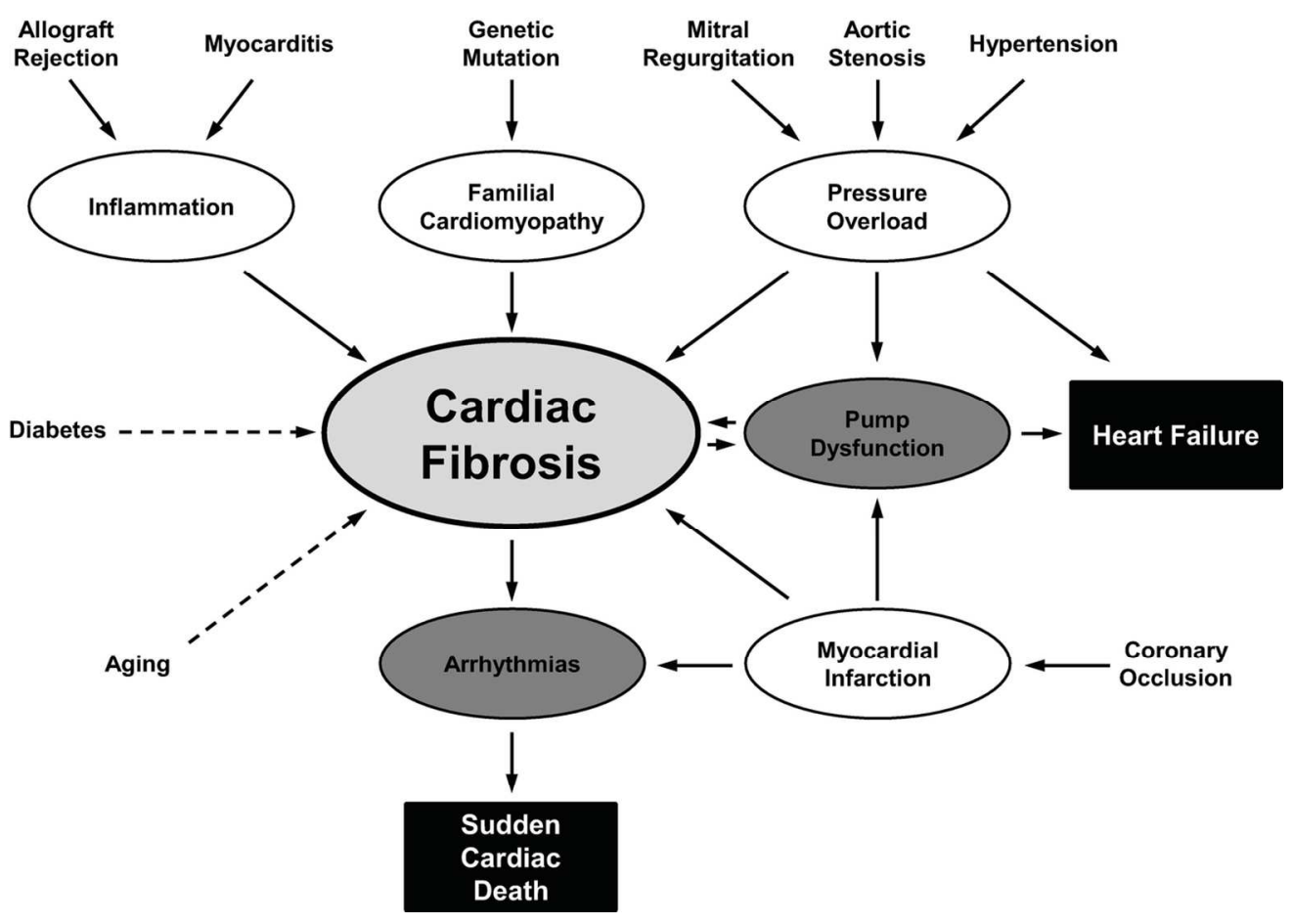

Figure 1

$123 \times 87 \mathrm{~mm}(300 \times 300 \mathrm{DPI})$ 


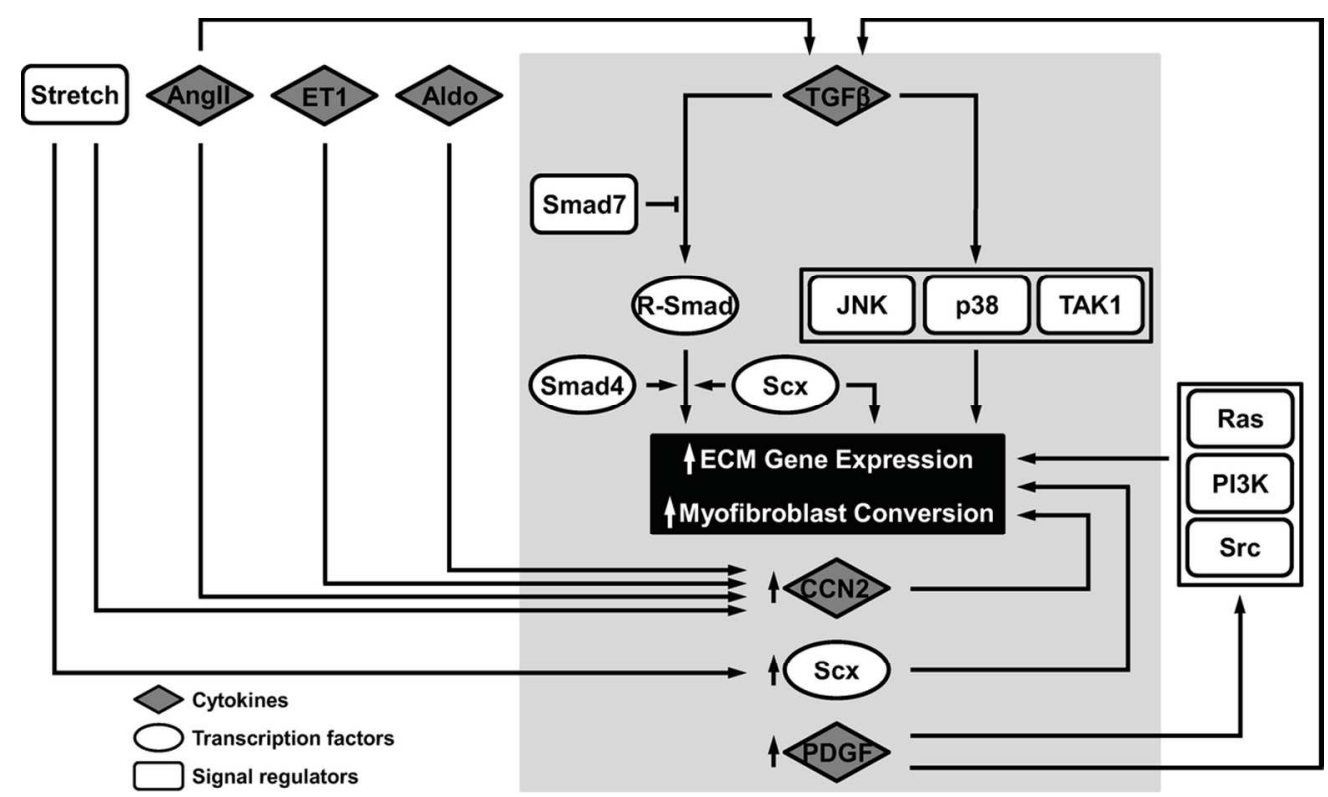

Figure 2

$105 \times 62 \mathrm{~mm}(300 \times 300 \mathrm{DPI})$ 